\title{
ON CYCLIC ADDITIVITY THEOREMS
}

BY

\author{
E. J. MICKLE AND T. RADO
}

\section{INTRODUCTION}

1. In this paper the topological structure theory of Peano spaces is applied to certain metric problems that arise in the theory of surface area. As a general reference for the many topological concepts involved, we use the book of G. T. Whyburn on Analytic topology (see the Bibliography at the end of this paper). For concepts and results relating to surface area theory, we use as a general reference the book of T. Rado on Length and area (see Bibliography), which contains also most of the topological information needed in the sequel. This book will be referred to as LA. Numbers in square brackets refer to literature cited in the Bibliography at the end of the paper.

2. Let $P$ be a Peano space, $P^{*}$ a metric space, and $T$ a continuous mapping from $P$ into $P^{*}$. To motivate the general study undertaken in this paper, let us first assume that $P^{*}$ is the Euclidean three-space, and that $P$ is either a 2-cell or a 2-sphere. Then $T$ may be thought of as a representation of an $F$-surface of the type of the 2-cell or the 2-sphere respectively (see LA II.3.44). Let us denote by $A(T)$ the Lebesgue area of this surface (see LA V.2.3). Then $A(T)$ may be thought of as a functional of the continuous mapping $T$. This functional has remarkable additivity properties. Let us consider a monotone-light factorization

$$
T=L M, \quad M: P \rightarrow \mathfrak{M}, \quad L: \mathfrak{M} \rightarrow P^{*}
$$

of $T$ (see LA II.1.18), where $\rightarrow$ indicates a mapping onto, $\rightarrow$ indicates a mapping into, and products of mappings are read from the right to the left. Let $C$ be a generic notation for a proper cyclic element of the middle-space $\mathfrak{M}$, and let $r_{C}$ denote the monotone retraction from $\mathfrak{M}$ onto $C$. We have then the remarkable formula

$$
A(T)=\sum A\left(L r_{C} M\right)
$$

where the summation is extended over all the proper cyclic elements of $\mathfrak{M}$. The reader is referred to LA II.5.10 for historical remarks and technical comments concerning the work of C. B. Morrey, J. W.T. Youngs, and T. Rado relating to the above formula (see also Bibliography, [2], [3], [4]).

We shall refer to the property of $A(T)$, expressed by the above formula, as weak additivity, in contradistinction with a stronger property that we shall term strong additivity. Our search for this strong additivity property was sug-

Presented to the Society, April 16, 1948; received by the editors April 27, 1948. 
gested by the study of a paper of J. W. T. Youngs, as yet unpublished, on the isoperimetric inequality. In that paper, J. W. T. Youngs developed a general additivity theorem for the homomorphisms induced by continuous mappings between the cohomology groups of compact metric spaces. In the additivity theorem for such homomorphisms, he considered factorizations that were not assumed to be monotone-light. While studying his work, we raised the question whether a similar strong additivity theorem holds for the Lebesgue area $A(T)$. The affirmative answer to this question is one of the results established in the present paper. The following comments concerning our method may be of interest. To establish the strong additivity (see \$4.1) of the Lebesgue area $A(T)$, it would be natural to follow the pattern of proof employed in [4] to establish the weak additivity. In the paper just quoted, the weak additivity is proved for a whole class of functionals possessing certain properties. The application to the Lebesgue area is then made by showing that the Lebesgue area $A(T)$ possesses the properties required for the validity of the general weak additivity theorem (see LA V.2.55). Similarly, in the present paper we first derive a strong additivity theorem for general functionals (see part 4), and the application to the Lebesgue area could be made by showing that $A(T)$ possesses the properties required for the validity of our strong additivity theorem. This would require a laborious study of metric properties of $A(T)$ which we omit because we have a far more elegant and instructive method at our disposal. Indeed, in part 5 we show that under certain very general conditions the strong additivity property is an essential topological consequence of the weak additivity property. The application to the Lebesgue area is immediate (see $\$ 5.6$ ).

\section{Definitions AND lemmas}

1.1. Throughout Part $1, P$ is a fixed Peano space and $P^{*}$ is a fixed metric space (see LA I.2.10, I.2.33). $\mathcal{T}$ denotes the class of all continuous mappings $T$ from $P$ into $P^{*}$. Thus an inclusion $T \in \mathcal{T}$ means that $T$ is defined and continuous on $P$ and for every point $p \in P$ the image point $T(p)=p^{*}$ lies in $P^{*}$.

1.2. Given three mappings $T, T_{1}, T_{2}$ in $\mathcal{T}$, we shall say that $T_{1}, T_{2}$ constitute a partition of $T$ if there exists a point $p_{0}^{*} \in P^{*}$ and a pair of subsets $E_{1}, E_{2}$ in $P$ such that the following conditions hold. (a) $E_{1}, E_{2}$ are closed sets. (b) $E_{1}+E_{2}=P$. (c) $T_{1}, T_{2}$ satisfy the relations

$$
T_{1}(p)=\left\{\begin{array}{ll}
T(p) & \text { if } \quad p \in E_{1}, \\
p_{0}^{*} & \text { if } \quad p \in E_{2},
\end{array} \quad T_{2}(p)=\left\{\begin{array}{lll}
p_{0}^{*} & \text { if } & p \in E_{1}, \\
T(p) & \text { if } & p \in E_{2} .
\end{array}\right.\right.
$$

1.3. An unrestricted factorization of a mapping $T \in \mathcal{T}$ consists of two mappings $f, s$ and of a Peano space $\mathfrak{M}$, such that the following conditions hold.

(a) $f$ is a continuous mapping from $P$ into $\mathfrak{M}$.

(b) $s$ is a continuous mapping from $\mathfrak{M}$ into $P^{*}$.

(c) $T=s f$. 
The following remarks are in order. As regards (c), let us recall the agreement that product mappings are to be read from the right to the left throughout the paper. As regards $\mathfrak{M}$, it may be any Peano space. In particular, the image $f(P)$ may be a proper subset of $\mathfrak{M}$. An unrestricted factorization will be indicated by writing

$$
T=s f, \quad f: P \rightarrow \mathfrak{M}, \quad s: \mathfrak{M} \rightarrow P^{*} .
$$

The mappings $f, s$ will be termed the first and the second factor respectively, while $\mathfrak{M}$ will be called the middle-space of the unrestricted factorization.

1.4. The term unrestricted factorization is used to stress the generality of the situation as compared with the so-called monotone-light factorizations. In a monotone-light factorization, the first factor $f$ is required to be monotone, the second factor $s$ is required to be light, and $f$ is required to map $P$ onto $\mathfrak{M}$ (see LA II.1.18). The last condition places a severe restriction upon $\mathfrak{M}$. For example, if $P$ is a 2 -sphere, then the middle-space occurring in a monotonelight factorization must be a cactoid.

1.5. Given $T \in \mathcal{T}$, consider an unrestricted factorization $T=s f, f: P \rightarrow \mathfrak{M}$, $s: \mathfrak{M} \rightarrow P^{*}$. Let $A, A_{1}, A_{2}$ be $A$-sets in $\mathfrak{M}$ (see LA II.2.31) such that

$$
A_{1}+A_{2}=A, \quad A_{1} A_{2}=\mathfrak{x}_{0},
$$

where $\mathfrak{x}_{0}$ is a single point. Let $r, r_{1}, r_{2}$ be the monotone retractions from $\mathfrak{M}$ onto $A, A_{1}, A_{2}$ respectively (see LA II.2.40, II.2.43). Let us denote by $\bar{r}_{1}$ the monotone retraction from $A$ onto $A_{1}$ and by $\bar{r}_{2}$ the monotone retraction from $A$ onto $A_{2}$. We have then the formulas (see LA II.2.100)

$$
r_{1}=\bar{r}_{1} r, \quad r_{2}=\bar{r}_{2} r \text {. }
$$

An easy argument shows that $r_{1}=\bar{r}_{1}, r_{2}=\bar{r}_{2}$ on $A$ (cf. LA II.2.42, II.2.43, II.2.81). There follow the formulas

$$
r_{1}=r_{1} r, \quad r_{2}=r_{2} r .
$$

Let us introduce the mappings

$$
\tau=s r f, \quad \tau_{1}=s r_{1} f, \quad \tau_{2}=s r_{2} f .
$$

Then clearly $\tau, \tau_{1}, \tau_{2}$ belong to $\mathcal{T}$.

LEMMA. The mappings $\tau_{1}, \tau_{2}$ constitute a partition of $\tau$ (see 1.2).

Proof. Let us define the point $p_{0}^{*}$ and the sets $E_{1}, E_{2}$ by the formulas (see (1)):

$$
\begin{aligned}
& p_{0}^{*}=s\left(\mathfrak{x}_{0}\right) . \\
& E_{1}=(r f)^{-1}\left(A_{1}\right), \quad E_{2}=(r f)^{-1}\left(A_{2}\right) .
\end{aligned}
$$

In view of (2), we have 


$$
\left(r_{1} f\right)(p)=\left(r_{1} r f\right)(p)=r_{1}[(r f)(p)]
$$

If $p \in E_{1}$, then $(r f)(p) \in A_{1}$ by (5), and hence by (6)

$$
\left(r_{1} f\right)(p)=(r f)(p)
$$

if $p \in E_{1}$,

since $r_{1}(\mathfrak{x})=\mathfrak{x}$ if $\mathfrak{x} \in A_{1}$. On the other hand, if $p \in E_{2}$, then $(r f)(p) \in A_{2}$ by (5), and hence by (6)

$$
\left(r_{1} f\right)(p)=\mathfrak{x}_{0}
$$

if $p \in E_{2}$,

since clearly $r_{1}(\mathfrak{x})=\mathfrak{x}_{0}$ if $\mathfrak{x} \in A_{2}$ (cf. LA II.2.40). From (3), (7), (4) we infer that

$$
\tau_{1}(p)= \begin{cases}\tau(p) & \text { if } p \in E_{1}, \\ p_{0}^{*} & \text { if } p \in E_{2} .\end{cases}
$$

A similar reasoning shows that

$$
\tau_{2}(p)= \begin{cases}p_{0}^{*} & \text { if } p \in E_{1}, \\ \tau(p) & \text { if } p \in E_{2} .\end{cases}
$$

In view of (5) and (1) it is clear that $E_{1}, E_{2}$ are closed and $E_{1}+E_{2}=P$. Thus $\tau_{1}, \tau_{2}$ constitute a partition of $\tau$, in the sense of 1.2.

1.6. Let $\Phi(T)$ be a functional, defined for $T \in \mathcal{T}$, satisfying the following conditions.

(a) $\Phi(T)$ is real-valued and non-negative. For certain mappings $T \in \mathcal{T}$, we may have $\Phi(T)=+\infty$.

(b) If $T$ is constant on $P$, then $\Phi(T)=0$.

(c) $\Phi(T)$ is l.s.c. (lower semi-continuous), in the following sense. Given two mappings $T^{\prime}, T^{\prime \prime}$ in $\mathcal{T}$, their distance $d\left(T^{\prime}, T^{\prime \prime}\right)$ is defined as $\max \rho\left[T^{\prime}(p), T^{\prime \prime}(p)\right], p \in P$, where $\rho$ denotes the distance in the metric space $P^{*}$ (note that the use of the maximum is justified since $P$ is compact). The requirement of lower semi-continuity means that if $T_{n} \in \mathcal{T}, n=0,1,2, \cdots$, and $d\left(T_{n}, T_{0}\right) \rightarrow 0$ for $n \rightarrow \infty$, then $\Phi\left(T_{0}\right) \leqq \lim \inf \Phi\left(T_{n}\right)$.

(d) $\Phi$ is additive under partition, in the following sense. If the mappings $T_{1}, T_{2}$ form a partition of the mapping $T$ (see 1.2), then $\Phi(T)=\Phi\left(T_{1}\right)+\Phi\left(T_{2}\right)$.

1.7. Given a functional $\Phi$ with the properties stated in 1.6 , let $T=s f$, $f: P \rightarrow \mathfrak{M}, s: \mathfrak{M} \rightarrow P^{*}$, be an unrestricted factorization of a mapping $T \in \mathcal{G}$. Let $A_{1}, \cdots, A_{n}$ be $A$-sets in $\mathfrak{M}$ such that $A_{1}+\cdots+A_{n}=\mathfrak{M}$ and for each $j=1, \cdots, n-1$ the product $\left(A_{1}+\cdots+A_{j}\right) A_{j+1}$ is a single point $\mathfrak{x}_{j}$. Finally, let $r_{j}$ denote the monotone retraction from $\mathfrak{M}$ onto $A_{j}, j=1, \cdots n$.

LEMMA. Under the conditions just stated, we have the formula

$$
\Phi(T)=\sum_{j=1}^{n} \Phi\left(s r_{j} f\right)
$$


Proof. Proceeding by induction, we assume first that $n=2$. By the lemma of 1.5 , the mappings $s r_{1} f, s r_{2} f$ constitute a partition of $T$ (in the present case, $A_{1}+A_{2}=\mathfrak{M}$ and hence the mapping $r$ of 1.5 reduces now to the identity). Thus, for $n=2$, the formula (1) follows directly from 1.6 (d). Let us assume that the lemma has been proved for the case when the number of the $A$-sets involved is not greater than $n-1$. To establish (1), we write

$$
\mathfrak{M}=\left(A_{1}+\cdots+A_{n-1}\right)+A_{n} .
$$

Then $A_{1}+\cdots+A_{n-1}$ is again an $A$-set, and $\left(A_{1}+\cdots+A_{n-1}\right) A_{n}$ is a single point. Since we already verified the lemma for the case of two $A$-sets, there follows the formula

$$
\Phi(T)=\Phi(s r f)+\Phi\left(s r_{n} f\right),
$$

where $r$ denotes the monotone retraction from $\mathfrak{M}$ onto $A_{1}+\cdots+A_{n-1}$. For the mapping $\operatorname{srf}$ we have the unrestricted factorization

(3) $\quad s r f=s(r f), \quad r f: P \rightarrow A_{1}+\cdots+A_{n-1}, \quad s: A_{1}+\cdots+A_{n-1} \rightarrow P^{*}$.

Now each $A_{j}, j=1, \cdots, n-1$, is an $A$-set relative to $A_{1}+\cdots+A_{n-1}$ (see LA II.2.81). Since the lemma is assumed to hold for the case of $n-1 A$-sets, we infer from (3) the formula

$$
\Phi(s r f)=\sum_{j=1}^{n-1} \Phi\left(s \bar{r}_{j} r f\right),
$$

where $\bar{r}_{j}$ is the monotone retraction from $A_{1}+\cdots+A_{n-1}$ onto $A_{j}$. Clearly $\bar{r}_{j} r$ is a monotone retraction from $\mathfrak{M}$ onto $A_{j}$, and hence $\bar{r}_{j} r=r_{j}$ (see LA II.2.43). Thus (4) yields

$$
\Phi(s r f)=\sum_{j=1}^{n-1} \Phi\left(s r_{j} f\right)
$$

Finally, (1) follows from (2) and (5).

1.8. Given a functional $\Phi$ with the properties stated in 1.6 , let $T=s f$, $f: P \rightarrow \mathfrak{M}, s: \mathfrak{M} \rightarrow P^{*}$, be an unrestricted factorization of a mapping $T \in \mathcal{T}$. Let $A$ be an $A$-set in $\mathfrak{M}$, and let $r$ be the monotone retraction from $\mathfrak{M}$ onto $A$.

LEMмA. Under the conditions just stated, we have the inequality

$$
\Phi(T) \geqq \Phi(s r f) \text {. }
$$

Proof. Case (a). $A=\mathfrak{M}$. Then $r$ reduces to the identity, and (1) is obvious.

Case (b). $A$ is a proper subset of $\mathfrak{M}$, and the set $\mathfrak{M}-A$ has a finite number of components $S_{1}, \cdots, S_{n}$. If we denote by $A_{j}$ the closure of $S_{j}$, then $A_{j}$ is an $A$-set (see LA II.2.73), and $A+A_{1}+\cdots+A_{n}=\mathfrak{M}$. Furthermore, each one of the products $A A_{1},\left(A+A_{1}\right) A_{2}, \cdots,\left(A+A_{1}+\cdots+A_{n-1}\right) A_{n}$ reduces to a single point (see LA II.2.35, II.2.73). Hence, by the lemma in 1.7, 


$$
\Phi(T)=\Phi(s r f)+\sum_{j=1}^{n} \Phi\left(s r_{j} f\right)
$$

where $r_{j}$ is the monotone retraction from $\mathfrak{M}$ onto $A_{j}$. Since $\Phi$ is non-negative, (2) implies (1).

Case (c). $A$ is a proper subset of $\mathfrak{M}$, and the set $\mathfrak{M}-A$ has infinitely many components $S_{1}, \cdots, S_{n}, \cdots$. In this case, let us put

$$
A_{n}=A+\sum_{j=n+1}^{\infty} S_{j} .
$$

Then $A_{n}$ is again an $A$-set of $\mathfrak{M}$, and the components of $\mathfrak{M}-A_{n}$ are precisely $S_{1}, \cdots, S_{n}$ (see LA II.2.75). Thus we can apply the result established under case (b) to $A_{n}$. Hence, if we denote by $\bar{r}_{n}$ the monotone retraction from $\mathfrak{M}$ onto $A_{n}$, we have the inequality

$$
\Phi(T) \geqq \Phi\left(s \bar{r}_{n} f\right) .
$$

Now the diameter of $S_{j}$ converges to zero as $j \rightarrow \infty$ (see LA II.2.37). It follows that $\bar{r}_{n}$ converges, uniformly on $\mathfrak{M}$, to the monotone retraction $r$ from $\mathfrak{M}$ onto $A$ (cf. LA II.2.40). Hence $s \bar{r}_{n} f$ converges to $s r f$ uniformly on $\mathfrak{M}$. By 1.6 (c) it follows that

$$
\lim \inf \Phi\left(s \bar{r}_{n} f\right) \geqq \Phi(s r f) \quad \text { for } n \rightarrow \infty .
$$

Finally, (4) and (5) yield (1).

1.9. Let $T=s f, f: P \rightarrow \mathfrak{M}, s: \mathfrak{M} \rightarrow P^{*}$ be an unrestricted factorization of a mapping $T \in \mathcal{T}$. Let $A_{1}, \cdots, A_{j}, \cdots$ be a (finite or infinite) sequence of cyclic chains in $\mathfrak{M}$ that constitute a decomposition of $\mathfrak{M}$ in the sense of the cyclic chain approximation theorem (see LA II.2.71). Let $r_{j}$ be the monotone retraction from $\mathfrak{M}$ onto $A_{j}$. Finally, let $\Phi$ be a functional with the properties listed in 1.6.

Lемма. Under the conditions just stated, we have the formula

$$
\Phi(T)=\sum_{j} \Phi\left(s r_{j} f\right) .
$$

Proof. Case (a). The sequence $A_{1}, \cdots, A_{j}, \cdots$ is finite. Let it contain $n$ elements $A_{1}, \cdots, A_{n}$. Then $A_{1}, \cdots, A_{n}$ satisfy the assumptions of 1.7 , and thus (1) follows directly from the lemma proved there (cf. LA II.2.71).

Case (b). The sequence $A_{1}, \cdots, A_{n}, \cdots$ is infinite. Let us put

$$
\mathfrak{A}_{n}=A_{1}+\cdots+A_{n} \text {. }
$$

Then $\mathfrak{A}_{n}$ is an $A$-set, and hence there exists a unique monotone retraction $\bar{r}_{n}$ from $\mathfrak{M}$ onto $\mathfrak{A}_{n}$. If we denote by $d_{n}$ the maximum diameter of the components of the set $\mathfrak{M}-\mathfrak{A}_{n}$, then $d_{n} \rightarrow 0$ (see LA II.2.72). As a consequence, $\vec{r}_{n}$ converges uniformly to the identity on $\mathfrak{M}$ for $n \rightarrow \infty$, and hence $s \bar{r}_{n} f$ converges 
uniformly to $s f=T$. By 1.6 (c) there follows the inequality

$$
\Phi(T) \leqq \lim \inf \Phi\left(s \bar{r}_{n} f\right)
$$

for $n \rightarrow \infty$.

For the mapping $T_{n}=s \bar{r}_{n} f$ we have the unrestricted factorization

$$
T_{n}=s\left(\bar{r}_{n} f\right), \quad \bar{r}_{n} f: P \rightarrow \mathfrak{A}_{n}, \quad s: \mathfrak{A}_{n} \rightarrow P^{*} .
$$

In view of (2), the middle space $\mathfrak{A}_{n}$ presents case (a), already discussed. Hence, if we denote by $r_{j}^{n}$ the monotone retraction from $\mathfrak{A}_{n}$ onto $A_{j}$, $j=1, \cdots, n$, we have the formula

$$
\Phi\left(s \bar{r}_{n} f\right)=\sum_{j=1}^{n} \Phi\left(s r_{j}^{n} \bar{r}_{j} f\right) .
$$

Now clearly $r_{j}^{n} \bar{r}_{j}$ is a monotone retraction from $\mathfrak{M}$ onto $A_{j}$, and hence (see LA II.2.43)

$$
r_{j}^{n} \bar{r}_{j}=r_{j} .
$$

From (5), (4), (3) we infer the inequality

$$
\Phi(T) \leqq \sum_{j=1}^{\infty} \Phi\left(s r_{j} f\right) .
$$

To obtain the complementary inequality, we note that

$$
\Phi(T) \geqq \Phi\left(s \bar{r}_{n} f\right)
$$

by 1.8. From (4), (5), (7) there follows, for $n \rightarrow \infty$, the inequality

$$
\Phi(T) \geqq \sum_{j=1}^{\infty} \Phi\left(s r_{j} f\right) .
$$

Finally, (6) and (8) yield (1).

1.10. In 1.6 we permitted the functional $\Phi$ to take on the value $+\infty$ for certain mappings $T \in \mathcal{T}$. Hence a formula like 1.9 (1) is to be interpreted properly in cases where some of the $\Phi$-values involved are infinite. The plausible agreements that we used implicitly are as follows. If $q_{n}$ is a sequence of non-negative numbers, some of which may be infinite, then the formula

$$
q=\sum_{j} q_{j}
$$

has the following meaning. If at least one $q_{j}$ is infinite, then $q=+\infty$. If each $q_{j}$ is finite, but the series in (1) fails to converge, then $q=+\infty$. Finally, if each $q_{j}$ is finite and the series in (1) converges, then $q$ is equal to its sum.

\section{Monotone RETRACTIONS}

2.1. In the present part 2 we shall give some properties of monotone re- 
tractions and classes of monotone retractions defined on Peano spaces. Many of the results are well known but are included for the sake of completeness. Throughout part 2, $P$ will be a fixed Peano space. A continuous mapping $\tau$ from $P$ onto a Peano space $P_{0}$ will be indicated by writing $\tau: P \rightarrow P_{0}$, where the bold-face arrow means that the mapping is onto.

2.2. Lemma. Let $M: P \rightarrow \mathfrak{M}$ be a monotone mapping from $P$ onto a Peano space $\mathfrak{M}$. For every $A$-set $A$ in $P$ we have the following conditions satisfied. (a) $M$ is monotone on $A$. (b) $\mathfrak{A}=M(A)$ is either an $A$-set or a single point. (c) For the monotone retractions $r_{A}$ from $P$ onto $A$ and $r_{\mathfrak{A}}$ from $\mathfrak{M}$ onto $\mathfrak{A}, M r_{A}=r_{\mathfrak{A}} M$.

Proof. (a) Let $\mathfrak{x}_{0}$ be a point in $\mathfrak{A}=M(A)$. Since $M$ is monotone, $M^{-1}\left(\mathfrak{x}_{0}\right)$ is a connected set. Since $A$ is an $A$-set, $A M^{-1}\left(\mathfrak{x}_{0}\right)$ is a connected set (see LA II.2.42). Therefore $M$ is monotone on $A$.

(b) Assume that $\mathfrak{A}=M(A)$ is not an $A$-set or a single point. Then we have in $\mathfrak{M}$ a simple arc $\gamma$ with end points $\mathfrak{x}_{1}, \mathfrak{x}_{2} \in \mathfrak{A}$ such that $\gamma \mathfrak{A}=\mathfrak{x}_{1}+\mathfrak{x}_{2}$. Then $0 \neq A M^{-1}(\gamma)$ is a connected set. Hence $M\left[A M^{-1}(\gamma)\right]$ is a connected subset of $\gamma \mathfrak{A}=\mathfrak{x}_{1}+\mathfrak{x}_{2}$ and $M\left[A M^{-1}(\gamma)\right]$ contains $\mathfrak{x}_{1}$ and $\mathfrak{x}_{2}$. This is impossible. Therefore $\mathfrak{A}$ is an $A$-set.

(c) For the monotone mappings $M r_{A}$ and $r \mathscr{q} M$ we have that

$$
M r_{A}(p)=r_{\mathfrak{A}} M(p)=M(p) \quad \text { for } p \in A \text {. }
$$

If $A=P$, then the proof of (c) follows from (1). Assume that $A \neq P$. Let $G$ be a component of $P-A$. Since $A$ is an $A$-set, the frontier of $G$ is a single point $p_{0}$ (see LA II.2.35). Then (see LA II.2.40, II.2.43)

$$
M r_{A}(p)=M\left(p_{0}\right)
$$

for $p \in G$.

We assert that

$$
r(p)=M\left(p_{0}\right) \quad \text { for } p \in G \text {. }
$$

Set $M_{0}=r_{\mathfrak{A}} M$ and let $p$ be a point in $G$. Then $M_{0}(p) \in \mathfrak{A}$ and $M_{0}^{-1}\left[M_{0}(p)\right]$ is a connected set whose intersection with $A$ and with $G$ is non-empty. Hence $p_{0} \in M_{0}^{-1}\left[M_{0}(p)\right]$. Thus (3) holds. From (1), (2), and (3) it follows that $M r_{A}(p)=r \mathfrak{x} M(p)$ for $p \in P$.

2.3. Lemma. Let $M: P \rightarrow \mathfrak{M}$ be a monotone mapping from $P$ onto a Peano space $\mathfrak{M}$. If $M$ is light on a proper cyclic element $C$ of $P$, then $\mathfrak{C}=M(C)$ is a proper cyclic element of $\mathfrak{M}$ and for the monotone retractions $r_{C}$ from $P$ onto $C$ and r๕from $\mathfrak{M}$ onto $\mathfrak{C}, M r_{C}=r \Subset M$.

Proof. Since $C$ is a proper cyclic element of $P, C$ is an $A$-set (see LA II.2.50). Since $M$ is light on $C$, $\mathbb{E}$ is not a single point and hence, by (b) of 2.2, $\mathcal{C}$ is an $A$-set. By (a) of 2.2, $M$ is monotone on $C$ and, since $M$ is also light on $C$ by assumption, $M$ is topological on $C$. Hence $\mathbb{S}$ is cyclic. Therefore, since $\mathfrak{C}$ is an $A$-set which is cyclic, $\mathfrak{S}$ is a proper cyclic element of $\mathfrak{M}$ (see 
LA II.2.45). By (c) of 2.2, $M r_{C}=r \Subset M$.

2.4. Lemma. Let $M: P \rightarrow \mathfrak{M}$ be a monotone mapping from $P$ onto a Peano space $\mathfrak{M}$. If $\mathfrak{M}$ contains two distinct points $\mathfrak{x}_{1}$ and $\mathfrak{x}_{2}$ which are conjugate (see LA II.2.3), then there are points $p_{1} \in M^{-1}\left(\mathfrak{x}_{1}\right)$ and $p_{2} \in M^{-1}\left(\mathfrak{x}_{2}\right)$ such that $p_{1}$ and $p_{2}$ are conjugate.

Proof. Assume that $\mathfrak{M}$ contains two distinct points $\mathfrak{x}_{1}$ and $\mathfrak{x}_{2}$ which are conjugate. We have in $P$ a simple arc $\gamma$ with end points $p_{1} \in M^{-1}\left(\mathfrak{x}_{1}\right)$ and $p_{2} \in M^{-1}\left(\mathfrak{x}_{2}\right)$ such that $\gamma\left[M^{-1}\left(\mathfrak{x}_{1}\right)+M^{-1}\left(\mathfrak{x}_{2}\right)\right]=p_{1}+p_{2}$. Assume that $p_{1}$ and $p_{2}$ are not conjugate. Then there is a point $p_{0}$ such that $p_{1}, p_{2}$ are in different components of $P-p_{0}$. $p_{0}$ must necessarily be on $\gamma-\left(p_{1}+p_{2}\right)$ and hence $\mathfrak{x}_{0}=M\left(p_{0}\right) \neq \mathfrak{x}_{1}, \mathfrak{x}_{2}$. Since $\mathfrak{x}_{1}$ and $\mathfrak{x}_{2}$ are conjugate there is an arc $\gamma^{*}$ with end points $\mathfrak{x}_{1}, \mathfrak{x}_{2}$ such that $\gamma^{*}$ does not contain the point $\mathfrak{x}_{0}$. Then $M^{-1}\left(\gamma^{*}\right)$ is a connected set and $p_{1}+p_{2} \subset M^{-1}\left(\gamma^{*}\right) \subset P-p_{0}$. Thus $p_{1}$ and $p_{2}$ must be in the same component of $P-p_{0}$. This is a contradiction. Hence $p_{1}$ and $p_{2}$ are conjugate.

2.5. Lemma. Let $M: P \rightarrow \mathfrak{M}$ be a monotone mapping from $P$ onto a Peano space $\mathfrak{M}$. If $M$ is constant on each proper cyclic element of $P$, then $\mathfrak{M}$ is a dendrite.

Proof. Assume that $\mathfrak{M}$ is not a dendrite. Then $\mathfrak{M}$ contains two distinct points $\mathfrak{x}_{1}, \mathfrak{x}_{2}$ which are conjugate. By 2.4 there are points $p_{1} \in M^{-1}\left(\mathfrak{x}_{1}\right)$, $p_{2} \in M^{-1}\left(\mathfrak{x}_{2}\right)$ such that $p_{1}, p_{2}$ are conjugate. Then $p_{1}, p_{2}$ belong to a proper cyclic element of $P$. This contradicts the assumption that $M$ is constant on each proper cyclic element of $P$. Therefore $\mathfrak{M}$ is a dendrite.

2.6. Lemma. Let $M: P \rightarrow \mathfrak{M}$ be a monotone mapping from $P$ onto a Peano space $\mathfrak{M}$. For each proper cyclic element $\mathfrak{S}$ of $\mathfrak{M}$ there is a proper cyclic element $C$ of $P$ such that $M(C) \supset \mathcal{S}$.

Proof. Assume that for a given proper cyclic element $\mathbb{C}$ of $\mathfrak{M}$ there is no proper cyclic element $C$ of $P$ such that $M(C) \supset$ S. For each proper cyclic element $C$ of $P, M(C)$ is either an $A$-set or a single point (see 2.2). Hence $M(C) \mathfrak{C}$ is either the empty set or a single point (see LA II.2.45). Let $r \Subset$ be the monotone retraction from $\mathfrak{M}$ onto $\mathfrak{C}$. Then $r \Subset M$ is a monotone mapping from $P$ onto $\mathfrak{E}$ and $r \Subset M$ is constant on each proper cyclic element of $P$. By 2.5 , $\mathfrak{S}$ is a dendrite. This contradicts the assumption that $\mathfrak{S}$ is a proper cyclic element of $\mathfrak{M}$. Therefore for each proper cyclic element $\mathfrak{S}$ of $\mathfrak{M}$ there is a proper cyclic element $C$ of $P$ such that $M(C) \supset \mathbb{E}$.

2.7. Let $T$ be a mapping in $\mathcal{\tau}$ (see 1.1) and let $T=L M, M: P \rightarrow \mathfrak{M}$, $L: \mathfrak{M} \rightarrow P^{*}$ be a monotone-light factorization of $T$ (see 1.4 ).

Let $C$ be a proper cyclic element of $P$ and let $r_{C}$ be the monotone retraction from $P$ onto $C . \delta(T)$ is defined to be the class of mappings $\operatorname{Tr}_{C}$ for all proper cyclic elements $C$ of $P$ on which $T$ is not constant. Note that $\delta(T)$ is a class of distinct mappings in $\mathcal{T}$. 
Let $\mathbb{S}$ be a proper cyclic element of the middle space $\mathfrak{M}$ of the monotonelight factorization of $T$ and let $r \mathfrak{E}$ be the monotone retraction from $\mathfrak{M}$ onto $\mathfrak{S}$. $\Delta(T)$ is defined to be the class of mappings $\operatorname{Lr} M$ for all proper cyclic elements $\mathfrak{C}$ of $\mathfrak{M}$. It should be noted that $\Delta(T)$ is a class of distinct mappings in $\mathcal{T}$ and that, by LA II.2.95, $\Delta(T)$ is independent of the choice of the monotone-light factorization of $T$.

Let $P_{0}$ be a Peano space and let $T_{0}: P_{0} \rightarrow P$ be a continuous mapping from $P_{0}$ onto $P . \delta(T) T_{0}$ is defined to be the class of mappings $\operatorname{Tr}_{C} T_{0}$ for all mappings $\operatorname{Tr}_{C}$ in $\delta(T)$ and $\Delta(T) T_{0}$ is defined to be the class of mappings $\operatorname{Lr} M T_{0}$ for all mappings $\operatorname{Lr} M$ in $\Delta(T)$. As a special case, it follows that $\Delta(T)=\delta(L) M$.

2.8. Lemma. Let $M_{0}: P_{0} \rightarrow P$ be a monotone mapping from a Peano space $P_{0}$ onto $P$ and let $T$ be a mapping in $\tau$ (see 1.1). Then $\Delta\left(T M_{0}\right)=\Delta(T) M_{0}$.

Proof. Let $T=L M, M: P \rightarrow \mathfrak{M}, L: \mathfrak{M} \rightarrow P^{*}$, be a monotone-light factorization of $T$. Then $T M_{0}=L\left(M M_{0}\right), M M_{0}: P_{0} \rightarrow \mathfrak{M}, L: \mathfrak{M} \rightarrow P^{*}$, is a monotone-light factorization of $T M_{0}$. Let $\mathfrak{E}$ be a proper cyclic element of $\mathfrak{M}$ and let $r \in \mathfrak{c}$ be the monotone retraction from $\mathfrak{M}$ onto $\mathbb{C}$. Then $\operatorname{Lr} \mathbb{E} M$ is in $\Delta(T)$ and $\operatorname{Lr} \mathbb{C} M M_{0}$ is in $\Delta\left(T M_{0}\right)$. Therefore $\Delta\left(T M_{0}\right)=\Delta(T) M_{0}$.

2.9. Lemma. Let $T$ be a mapping in $\mathcal{T}$ (see 1.1). Assume that the proper cyclic elements $C$ of $P$ can be divided into two classes $K_{1}, K_{2}$ such that $T$ is constant on each $C \in K_{1}$ and $T$ is light on each $C \in K_{2}$ (either $K_{1}$ or $K_{2}$ may be empty). Then $\delta(T)=\Delta(T)$.

Proof. Let $T=L M, M: P \rightarrow \mathfrak{M}, L: \mathfrak{M} \rightarrow P^{*}$, be a monotone-light factorization of $T$. Then $M$ is constant on each $C \in K_{1}$ and $M$ is light (in fact, topological) on each $C \in K_{2}$. For each $C \in K_{1}, M(C)$ is a point and hence $M(C)$ is not a proper cyclic element of $\mathfrak{M}$. By 2.3 , for each $C \in K_{2}, \mathfrak{S}=M(C)$ is a proper cyclic element of $\mathfrak{M}$ and by 2.6 the formula $\mathfrak{E}=M(C), C \in K_{2}$, gives all the proper cyclic elements of $\mathfrak{M}$. By $2.3, M r_{C}=r \Subset M$ for $C \in K_{2}, \mathfrak{S}=M(C)$. Hence $T r_{C}=L M r_{C}=L r \Subset M$ for each $C \in K_{2}, \mathbb{E}=M(C)$. Therefore $\delta(T)=\Delta(T)$.

\section{Partitions of MaPpings}

3.1. Throughout the present part $3, P$ is a fixed Peano space, $P^{*}$ is a fixed metric space, and $\mathcal{T}$ denotes the class of all continuous mappings from $P$ into $P^{*}$ (see 1.1).

3.2. LemMA. Let $T$ and $T_{0}$ be mappings in $\mathcal{T}$ and let $T=L M, M: P \rightarrow \mathbb{M}$, $L: \mathfrak{M} \rightarrow P^{*}$, be a monotone-light factorization of $T$ (see 1.4). Assume that $T_{0}$ is constant on each set $M^{-1}(\mathfrak{x})$ for $\mathfrak{x} \in \mathfrak{M}$. Then $\tau=T_{0} M^{-1}(\mathfrak{x})$ is a continuous mapping from $\mathfrak{M}$ into $P^{*}$ and $T_{0}=\tau M$.

Proof. Since $T_{0}$ is constant on each set $M^{-1}(\mathfrak{x}), \mathfrak{x} \in \mathfrak{M}, \tau=T_{0} M^{-1}(\mathfrak{x})$ is a single-valued mapping from $\mathfrak{M}$ into $P^{*}$ and $T_{0}=T_{0} M^{-1} M=\tau M$. The con- 
tinuity of $\tau$ then follows by an elementary reasoning.

3.3. Lemma. Under the conditions stated in 3.2 let $E_{1}$ be a set in $P$ on which $T_{0}(p)=T(p)$ and let $E_{2}$ be a set in $P$ on which $T_{\theta}(p)=p_{0}^{*}$. Then $\tau(\mathfrak{x})$ $=L(\mathfrak{x})$ for $\mathfrak{x} \in M\left(E_{1}\right)$ and $\tau(\mathfrak{x})=p_{0}^{*}$ for $\mathfrak{x} \in M\left(E_{2}\right)$.

Proof. If $\mathfrak{x} \in M\left(E_{1}\right)$ then $L(\mathfrak{x})=T M^{-1}(\mathfrak{x})$ and $\tau(\mathfrak{x})=T_{0} M^{-1}(\mathfrak{x})$. Since $M^{-1}(\mathfrak{x}) E_{1} \neq 0$ and $T$ and $T_{0}$ are constant on $M^{-1}(\mathfrak{x}), T_{0} M^{-1}(\mathfrak{x})=T M^{-1}(\mathfrak{x})$. Therefore $\tau(\mathfrak{x})=L(\mathfrak{x})$.

If $\mathfrak{x} \in M\left(E_{2}\right)$ then $M^{-1}(\mathfrak{x}) E_{2} \neq 0$. Since $T_{0}$ is constant on $M^{-1}(\mathfrak{x}), \tau(\mathfrak{x})$ $=T_{0} M^{-1}(\mathfrak{x})=p_{0}^{*}$.

3.4. Let $T_{1}, T_{2}$ be mappings in $\mathcal{T}$ which constitute a partition of a mapping $T$ in $\mathcal{T}$ (see 1.2). Then there exists a point $p_{0}^{*} \in P^{*}$ and a pair of subsets $E_{1}, E_{2}$ in $P$ such that the following conditions hold. (a) $E_{1}, E_{2}$ are closed sets. (b) $E_{1}+E_{2}=P$. (c) $T_{1}$ and $T_{2}$ satisfy the relations

$$
T_{1}(p)=\left\{\begin{array}{ll}
T(p) & \text { if } p \in E_{1}, \\
p_{0}^{*} & \text { if } p \in E_{2},
\end{array} \quad T_{2}(p)=\left\{\begin{array}{lll}
p_{0}^{*} & \text { if } p \in E_{1}, \\
T(p) & \text { if } p \in E_{2} .
\end{array}\right.\right.
$$

In the sequel we shall refer to this section for the notation for a partition of a mapping $T$ and also for the notation

$$
T=L M, \quad M: P \rightarrow \mathfrak{M}, \quad L: \mathfrak{M} \rightarrow P^{*},
$$

for a monotone-light factorization of $T$.

3.5. Lemma. Let a partition of a mapping $T \in \mathcal{T}$ be given as in 3.4. For each connected set $E$ in $P-T^{-1}\left(p_{0}^{*}\right)$ either (a) $E \subset E_{1}, E E_{2}=0$ or (b) $E \subset E_{2}, E E_{1}=0$.

Proof. Let $E$ be a connected set in $P-T^{-1}\left(p_{0}^{*}\right)$. Now $E E_{1}$ is the set of points of $E$ where $T_{2}(p)=p_{0}^{*}$ and $E E_{2}$ is the set of points of $E$ where $T_{1}(p)$ $=p_{0}^{*}$. Hence $E E_{1}, E E_{2}$ are disjoint sets, the sum of $E E_{1}$ and $E E_{2}$ is $E$, and $E E_{1}$, $E E_{2}$ are closed relative to $E$. Assume that $E E_{1} \neq 0$ and $E E_{2} \neq 0$. Then $E$ is not a connected set (see LA I.2.21). This is a contradiction. Hence either (a) $E E_{2}=0$ and $E \subset E_{1}$ or (b) $E E_{1}=0$ and $E \subset E_{2}$.

3.6. Lemma. Let a partition and a monotone-light factorization of a mapping $T \in \mathcal{T}$ be given as in 3.4. Then $T_{1}, T_{2}$ are constant on each set $M^{-1}(\mathfrak{x}), \mathfrak{x} \in \mathbb{M}$.

Proof. Let $\mathfrak{x}$ be a point in $\mathfrak{M}$. If $M^{-1}(\mathfrak{x}) \subset T^{-1}\left(p_{0}^{*}\right)$, then $T_{1}(p)=T_{2}(p)=p_{0}^{*}$ for $p \in M^{-1}(\mathfrak{x})$. If $M^{-1}(\mathfrak{x}) \subset P-T^{-1}\left(p_{0}^{*}\right)$, then, since $M^{-1}(\mathfrak{x})$ is a connected set, either (a) or (b) of 3.5 holds for $E=M^{-1}(\mathfrak{x})$. Hence both $T_{1}$ and $T_{2}$ are constant on $M^{-1}(\mathfrak{x})$.

3.7. LEMMA. Let a partition and a monotone-light factorization of a mapping $T \in \mathcal{T}$ be given as in 3.4. Set $\tau_{1}=T_{1} M^{-1}(\mathfrak{x}), \tau_{2}=T_{2} M^{-1}(\mathfrak{x}), \mathfrak{x} \in \mathfrak{M}$. Then $T_{1}=\tau_{1} M$, $T_{2}=\tau_{2} M$, and $\tau_{1}, \tau_{2}$ constitute $a$ partition of $L$.

Proof. By 3.6, $T_{1}$ and $T_{2}$ are constant on the sets $M^{-1}(\mathfrak{x}), \mathfrak{x} \in \mathfrak{M}$. By 3.2 
$\tau_{1}$ and $\tau_{2}$ are continuous mappings from $\mathfrak{M}$ into $P^{*}$ and $T_{1}=\tau_{1} M, T_{2}=\tau_{2} M$.

By 3.3 we have the following relations satisfied for the subsets $E_{1}, E_{2}$ described in 3.4 .

$$
\tau_{1}(\mathfrak{x})=\left\{\begin{array}{lll}
L(\mathfrak{x}) & \text { if } & \mathfrak{x} \in M\left(E_{1}\right), \\
p_{0}^{*} & \text { if } & \mathfrak{x} \in M\left(E_{2}\right),
\end{array} \quad \tau_{2}(\mathfrak{x})=\left\{\begin{array}{lll}
p_{0}^{*} & \text { if } & \mathfrak{x} \in M\left(E_{1}\right), \\
L(\mathfrak{x}) & \text { if } & \mathfrak{x} \in M\left(E_{2}\right) .
\end{array}\right.\right.
$$

Since $M\left(E_{1}\right)$ and $M\left(E_{2}\right)$ are closed sets whose sum is $\mathfrak{M}, \tau_{1}$ and $\tau_{2}$ constitute a partition of $L$ (see 1.2).

3.8. A Peano space $P_{0}$ will be said to possess the property $\lambda$ if the following condition is satisfied: For each proper cyclic element $C$ of $P_{0}$ and any closed and totally disconnected set $E$ in $C, C-E$ is a connected set.

As an example, a 2 -cell or a 2 -sphere possesses the property $\lambda$. Hence any monotone image of a 2 -cell or a 2 -sphere possesses the property $\lambda$.

3.9. Lemma. Let a partition of a mapping $T \in \mathcal{T}$ be given as in 3.4. Assume that the mapping $T$ is light and that $P$ possesses the property $\lambda$ (see 3.8). Then, for each proper cyclic element $C$ of $P$, either

$$
T_{1}(p)=T(p), \quad T_{2}(p)=p_{0}^{*}
$$

$$
\text { for } p \in C \text {, }
$$

or

$$
T_{2}(p)=T(p), \quad T_{1}(p)=p_{0}^{*}
$$$$
\text { for } p \in C \text {; }
$$

and (see 2.7) $\delta(T)=\delta\left(T_{1}\right)+\delta\left(T_{2}\right)$.

Proof. Let $C$ be a proper cyclic element of $P$. Since $P$ possesses the property $\lambda$ and $T$ is light, the set $E=C-T^{-1}\left(p_{0}^{*}\right)$ is a connected set in $P-T^{-1}\left(p_{0}^{*}\right)$. Hence either (a) or (b) of 3.5 holds. Since $T_{1}(p)=T_{2}(p)=T(p)$ $=p_{0}^{*}$ for $p \in T^{-1}\left(p_{0}^{*}\right)$, either (a) or (b) (and not both) of this section holds.

Let $K_{1}$ be the class of proper cyclic elements of $P$ where (a) holds and let $K_{2}$ be the class of proper cyclic elements of $P$ where (b) holds. Each proper cyclic element of $P$ is contained in either $K_{1}$ or $K_{2}$ and none is contained in both classes. Then (see 2.7) $\delta\left(T_{1}\right)$ is the class of mappings $T_{1} r_{C}=\operatorname{Tr}_{C}$ for $C \in K_{1}, \delta\left(T_{2}\right)$ is the class of mappings $T_{2} r_{C}=\operatorname{Tr}_{C}$ for $C \in K_{2}$, and $\delta(T)$ is the class of mappings $\operatorname{Tr}_{C}$ for $C \in K_{1}+K_{2}$. Therefore $\delta(T)=\delta\left(T_{1}\right)+\delta\left(T_{2}\right)$.

3.10. LemMA. Let a partition and a monotone-light factorization of a mapping $T \in \mathcal{T}$ be given as in 3.4 and let $\tau_{1}, \tau_{2}$ be the mappings defined in 3.7. Assume that the middle-space $\mathfrak{M}$ possesses the property $\lambda$ (see 3.8). Then for each proper cyclic element $\mathfrak{S}$ of $\mathfrak{M}$, either

$$
\tau_{1}(\mathfrak{x})=L(\mathfrak{x}), \quad \tau_{2}(\mathfrak{x})=p_{0}^{*}
$$

for $\mathfrak{x} \in \mathfrak{S}$,

or

$$
\tau_{2}(\mathfrak{x})=L(\mathfrak{x}), \quad \tau_{1}(\mathfrak{x})=p_{0}^{*}
$$

for $\mathfrak{x} \in \mathfrak{S}$;

and $\delta(L)=\delta\left(\tau_{1}\right)+\delta\left(\tau_{2}\right)$. 
Proof. By 3.7 the mappings $\tau_{1}, \tau_{2}$ constitute a partition of $L$. Since $\mathfrak{M}$, $L, \tau_{1}, \tau_{2}$ satisfy the conditions of 3.9 , the statements of 3.10 follow from 3.9.

3.11. THEOREM. Let a partition and a monotone-light factorization of a mapping $T \in \mathcal{T}$ be given as in 3.4. Assume that the middle-space $\mathfrak{M}$ possesses the property $\lambda$ (see 3.8). Then $\Delta(T)=\Delta\left(T_{1}\right)+\Delta\left(T_{2}\right)$.

Proof. Let $\tau_{1}, \tau_{2}$ be the mappings defined in 3.7. By 3.10

$$
\delta(L)=\delta\left(\tau_{1}\right)+\delta\left(\tau_{2}\right) .
$$

As noted in 2.7, $\Delta(T)=\delta(L) M$. Hence from (1)

$$
\Delta(T)=\delta\left(\tau_{1}\right) M+\delta\left(\tau_{2}\right) M .
$$

By (a) and (b) of $3.10, \tau_{1}, \tau_{2}$ are either light or constant on each proper cyclic element of $\mathfrak{M}$. By 2.9

$$
\Delta\left(\tau_{1}\right)=\delta\left(\tau_{1}\right), \quad \Delta\left(\tau_{2}\right)=\delta\left(\tau_{2}\right)
$$

and by 2.8

$$
\Delta\left(T_{1}\right)=\Delta\left(\tau_{1}\right) M, \quad \Delta\left(T_{2}\right)=\Delta\left(\tau_{2}\right) M .
$$

From (2), (3), and (4) it follows that $\Delta(T)=\Delta\left(T_{1}\right)+\left(T_{2}\right)$.

3.12. Lemma. Let a partition and a monotone-light factorization of a mapping $T \in \mathcal{T}$ be given as in 3.4. Assume that $\mathfrak{M}$ possesses the property $\lambda$. For each proper cyclic element (5) of $\mathfrak{M}$ either

$$
T_{1}(p)=T(p), \quad T_{2}(p)=p_{0}^{*} \quad \text { for } p \in M^{-1}(\mathfrak{S}),
$$

or

$$
T_{2}(p)=T(p), \quad T_{1}(p)=p_{0}^{*} \quad \text { for } p \in M^{-1}(\mathfrak{S}) .
$$

Proof. Let $\tau_{1}, \tau_{2}$ be the mappings defined in 3.7. By 3.7, $T_{1}=\tau_{1} M$ and $T_{2}=\tau_{2} M$. If (a) of 3.10 holds, then (a) of this section holds and if (b) of 3.10 holds, then (b) of this section holds.

3.13. Lemma. Assume that a mapping $T \in \mathcal{T}$ admits of an unrestricted factorization $T=s f, f: P \rightarrow \mathfrak{M}, s: \mathfrak{M} \rightarrow P^{*}$, where $\mathfrak{M}$ is a simple arc (see 1.3). Then, for any set $E$ in $P$ on which $T$ is not constant, there exist mappings $T_{1}$ and $T_{2}$ in $\mathcal{T}$ such that neither $T_{1}$ nor $T_{2}$ is constant on $E$ and $T_{1}, T_{2}$ constitute a partition of $T$.

Proof. Let $p_{1}, p_{2}$ be two points in $E$ such that $T\left(p_{1}\right) \neq T\left(p_{2}\right)$. Then $f\left(p_{1}\right) \neq f\left(p_{2}\right)$. Since $\mathfrak{M}$ is a simple arc there is a point $\mathfrak{x}_{0} \in \mathfrak{M}$ such that $s\left(\mathfrak{x}_{0}\right)$ $\neq s\left[f\left(p_{1}\right)\right], s\left[f\left(p_{2}\right)\right]$ and $\mathfrak{M}-\mathfrak{x}_{0}$ consists of two components $G_{1}, G_{2}$ with $f\left(p_{1}\right) \in G_{1}$ and $f\left(p_{2}\right) \in G_{2}$. Then $A_{1}=G_{1}+\mathfrak{x}_{0}$ and $A_{2}=G_{2}+\mathfrak{x}_{0}$ are $A$-sets and $A_{1} A_{2}=\mathfrak{x}_{0}$. Let $r_{1}$ be the monotone retraction from $\mathfrak{M}$ onto $A_{1}$ and let $r_{2}$ be the monotone retraction from $\mathfrak{M}$ onto $A_{2}$. By 1.5 the mappings $T_{1}=s r_{1} f, T_{2}=s r_{2} f$ 
are in $\mathcal{T}$ and constitute a partition of $T$. Now $T_{1}\left(p_{2}\right)=s\left(\mathfrak{x}_{0}\right) \neq T\left(p_{1}\right)=T_{1}\left(p_{1}\right)$ and $T_{2}\left(p_{1}\right)=s\left(\mathfrak{x}_{0}\right) \neq T\left(p_{2}\right)=T_{2}\left(p_{2}\right)$. Therefore neither $T_{1}$ nor $T_{2}$ is constant on $E$.

3.14. Theorem. Let $T$ be a mapping in $\mathcal{T}$ and let $T=L M, M: P \rightarrow \mathbb{M}$, $L: \mathfrak{M} \rightarrow P^{*}$, be a monotone-light factorization of $T$. Assume that $\mathfrak{M}$ possesses the property $\lambda$ and that $T$ admits of an unrestricted factorization in which the middle space is a simple arc. Then $\mathfrak{M}$ is a dendrite.

Proof. Assume that $\mathfrak{M}$ has a proper cyclic element $\mathfrak{E}$. Then $T$ is not constant on $M^{-1}(\mathfrak{E})$. Since $T$ admits of an unrestricted factorization in which the middle space is a simple arc, by 3.13 there exist mappings $T_{1}, T_{2} \in \mathcal{T}$ such that neither $T_{1}$ nor $T_{2}$ is constant on $M^{-1}(\mathfrak{E})$ and $T_{1}, T_{2}$ constitute a partition of $T$. Since $\mathfrak{M}$ posesses property $\lambda$, by 3.12 either $T_{1}$ or $T_{2}$ is constant on $M^{-1}(\mathfrak{E})$. These are contradictory statements and hence $\mathfrak{M}$ has no proper cyclic elements. Therefore $\mathfrak{M}$ is a dendrite.

\section{A GENERAL CYCLIC ADDITIVITY THEOREM}

4.1. Using the assumptions and notations of $1.1,1.3,1.6$, let $T=s f$, $f: P \rightarrow \mathfrak{M}, s: \mathfrak{M} \rightarrow P^{*}$, be an unrestricted factorization of a mapping $T \in \mathcal{T}$. If $C$ is a proper cyclic element of $\mathfrak{M}$ (see LA II.2.10, II.2.50), then $r_{C}$ will denote the monotone retraction from $\mathfrak{M}$ onto $C$. The functional $\Phi$ (see 1.6) is termed strongly additive if

$$
\Phi(T)=\sum \Phi\left(\operatorname{sr}_{C} f\right), \quad C \subset \mathfrak{M},
$$

for every $T \in \mathcal{T}$ and for every unrestricted factorization of $T$. In interpreting the preceding formula, the agreements stated in 1.10 are used, and it is understood that an empty summation has the value zero. Thus, in case $\mathfrak{M}$ has no proper cyclic elements, the formula means that $\Phi(T)=0$.

4.2. CyClic AdDITIVITy THEOREM. Suppose that the functional $\Phi$ satisfies the following condition in addition to those stated in 1.6.

(e) If the mapping $T \in \mathcal{T}$ admits of an unrestricted factorization where the middle space $\mathfrak{M}$ is a simple arc, then $\Phi(T)=0$.

Then $\Phi$ is strongly additive, in the sense of 4.1 .

The proof will be given in several steps.

4.3. Assume that the middle-space occurring in 4.1 (1) is a single point or a simple arc. Then the summation in 4.1 (1) is empty and hence its value is zero by agreement. On the other hand, $\Phi(T)=0$ by 1.6 (b) or 4.2 (e). Thus 4.1 (1) holds in these special cases.

4.4. Assume that the middle space $\mathfrak{M}$ occurring in $4.1(1)$ is a single cyclic chain $C\left(\mathfrak{x}_{1}, \mathfrak{x}_{2}\right)$ with a finite number of proper cyclic elements $C_{1}, \cdots, C_{n}$ (see LA II.2.21). From the structure of a cyclic chain (see LA II.2.63) there follows the existence of a finite cyclic chain decomposition $\mathfrak{M}=A_{1}+\cdots$ 
$+A_{m}$ (cf. 1.9, case (a)), such that each $A_{j}$ is either a simple arc or one of $C_{1}, \cdots, C_{n}$. Therefore, if we denote by $r_{j}$ the monotone retraction from $\mathfrak{M}$ onto $A_{j}$, we have by 1.9 the formula

$$
\Phi(T)=\sum_{j=1}^{m} \Phi\left(s r_{j} f\right)
$$

For the mapping $T_{j}=s r_{j} f$ we have the unrestricted factorization

$$
T_{j}=s\left(r_{j} f\right), \quad r_{j} f: P \rightarrow A_{j}, \quad s: A_{j} \rightarrow P^{*} .
$$

If $A_{j}$ is a simple arc, then $\Phi\left(T_{j}\right)=0$ by 4.2 (e). If $A_{j}$ is one of $C_{1}, \cdots, C_{n}$, say $A_{j}=C_{k}$, then $r_{j}=r_{C}$ for $C=C_{k}$. Thus (1) yields 4.1 (1).

4.5. Assume that the middle space $\mathfrak{M}$ occurring in 4.1 (1) is a single cyclic chain $C\left(\mathfrak{x}_{1}, \mathfrak{x}_{2}\right)$ with infinitely many proper cyclic elements $C_{1}, \cdots$, $C_{n}, \cdots$. Give any positive integer $j$ and consider $C_{1}, \cdots, C_{j}$. From the structure of a cyclic chain (see LA II.2.63) there follows the existence of a finite cyclic chain decomposition $\mathfrak{M}=A_{1}+\cdots+A_{m}$ (cf. 1.9, case (a)), such that each one of $C_{1}, \cdots, C_{j}$ coincides with one of $A_{1}, \cdots, A_{m}$. If we denote by $r$ the monotone retraction from $\mathfrak{M}$ onto $A_{k}$, then we have by 1.9 the formula

$$
\Phi(T)=\sum_{k=1}^{m} \Phi\left(s r_{k} f\right)
$$

If $\sum^{*}$ denotes summation restricted to those subscripts $k$ for which $A_{k}$ coincides with one of $C_{1}, \cdots, C_{j}$, then (1) yields (since $\Phi$ is non-negative) the inequality

$$
\Phi(T) \geqq \sum^{*} \Phi\left(s r_{k} f\right)=\sum_{n=1}^{j} \Phi\left(s r_{C_{n}} f\right) .
$$

Since $j$ is arbitrary, (2) yields

$$
\Phi(T) \geqq \sum \Phi\left(s r_{C} f\right),
$$

To derive the complementary inequality, we join the points $\mathfrak{x}_{1}, \mathfrak{x}_{2}$ of $\mathfrak{M}$ $=C\left(\mathfrak{x}_{1}, \mathfrak{x}_{2}\right)$ by a simple arc $c$. For each $n$, the intersection of $C_{n}$ and $c$ is a simple $\operatorname{arc} c_{n}$, and the $\operatorname{arcs} c_{1}, \cdots, c_{n}, \cdots$ have no interior points in common (see LA II.2.61). Since a simple arc is an absolute retract, we have for each $n$ a retraction $m_{n}$ (not necessarily monotone) from $C_{n}$ onto $c_{n}$. For each $j$, define a mapping

$$
\tau_{j}: \mathfrak{M} \rightarrow \mathfrak{M}_{j}=c+C_{1}+\cdots+C_{j}
$$

as follows: $\tau_{j}(\mathfrak{x})=\mathfrak{x}$ if $\mathfrak{x} \in \mathfrak{M}_{j}$, and $\tau_{j}(\mathfrak{x})=m_{n}(\mathfrak{x})$ if $\mathfrak{x} \in C_{n}, n>j$. Since the diameter of $C_{n}$ converges to zero as $n \rightarrow \infty$ (see LA II.2.52), $\tau_{j}$ is continuous on $\mathfrak{M}$ and is in fact a retraction (not necessarily monotone) from $\mathfrak{M}$ onto $\mathfrak{M}_{j}$. 
Furthermore, it follows that $\tau_{j}$ converges on $\mathfrak{M}$ uniformly to the identity, and hence $s \tau_{j} f$ converges uniformly to $s f=T$. By 1.6 (c) it follows that

$$
\Phi(T) \leqq \lim \inf \Phi\left(s \tau_{j} f\right)
$$

for $j \rightarrow \infty$.

Now $\mathfrak{M}_{j}$ is again a Peano space which is (relative to itself) a single cyclic chain, and the proper cyclic elements of $\mathfrak{M}_{j}$ are precisely $C_{1}, \cdots, C_{j}$ (see LA II.2.82). Hence, if we denote by $r_{j}^{\boldsymbol{k}}$ the monotone retraction from $\mathfrak{M}_{j}$ onto $C_{k}$, $k=1, \cdots, j$, we have by 4.4 the formula

$$
\Phi\left(s \tau_{j} f\right)=\sum_{k=1}^{j} \Phi\left(s r_{j}{ }_{j} j f\right) .
$$

In applying 4.4, we used here the unrestricted factorization

$$
s \tau_{j} f=s\left(\tau_{j} f\right), \quad \tau_{j} f: P \rightarrow \mathfrak{M}_{j}, \quad s: \mathfrak{M}_{j} \rightarrow P^{*} .
$$

Even though $\tau_{j}$ itself is not necessarily monotone, it follows readily that $r_{j}^{k} \tau_{j}$ is a monotone retraction from $\mathfrak{M}$ onto $C_{k}$, and hence (see LA II.2.43)

$$
\stackrel{k}{r_{j} \tau_{j}}=r_{C_{k}} \text {. }
$$

From (4), (5), (6) we infer the inequality

$$
\Phi(T) \leqq \sum \Phi\left(\operatorname{sr}_{C} f\right), \quad C \subset \mathfrak{M} .
$$

Finally, (3) and (7) yield 4.1 (1).

4.6. Assume finally that the middle space $\mathfrak{M}$ occuring in 4.1 (1) does not reduce to a single cyclic chain. Let then $A_{1}, \cdots, A_{n}, \cdots$ be a (finite or infinite) sequence of cyclic chains that constitute a decomposition of $\mathfrak{M}$ in the sense of the cyclic chain approximation theorem. By 1.9 we have

$$
\Phi(T)=\sum_{n} \Phi\left(s r_{n} f\right),
$$

where $r_{n}$ is the monotone retraction from $\mathfrak{M}$ onto $A_{n}$. For the mapping $s r_{n} f$ we have the unrestricted factorization

$$
s r_{n} f=s\left(r_{n} f\right), \quad r_{n} f: P \rightarrow A_{n}, \quad s: A_{n} \rightarrow P^{*} .
$$

By 4.3, 4.5 we have therefore

$$
\Phi\left(s r_{n} f\right)=\sum \Phi\left(s r^{n} r_{n} f\right), \quad C \subset A_{n},
$$

where $r_{C}^{n}$ is the monotone retraction from $A_{n}$ onto $C$. Now the proper cyclic elements of $A_{n}$ are precisely those proper cyclic elements of $\mathfrak{M}$ that are subsets of $A_{n}$ (see LA II.2.79). It follows that

$$
r^{n} r_{n}=r_{C}
$$

where $r_{C}$ is the monotone retraction from $\mathfrak{M}$ onto $C$. Furthermore, each proper cyclic element of $\mathfrak{M}$ is a subset of precisely one of the cyclic chains 
$A_{n}$. Thus (1), (2), (3) yield

$$
\Phi(T)=\sum \Phi\left(s r_{C} f\right), \quad C \subset \mathfrak{M},
$$

and the proof of the theorem stated in 4.2 is complete.

\section{A RELATIONSHIP BETWEEN CYCLIC ADDITIVITY THEOREMS}

5.1. Using the assumptions and notations of $1.1,1.4$, let $T=L M$, $M: P \rightarrow \mathfrak{M}, L: \mathfrak{M} \rightarrow P^{*}$, be a monotone-light factorization of a mapping $T \in \mathcal{G}$. If $C$ is a proper cyclic element of $\mathfrak{M}$ then $r_{C}$ will denote the monotone retraction from $\mathfrak{M}$ onto $C$. A functional $\Phi$ satisfying the conditions (a), (b), (c) of 1.6 is termed weakly additive if

$$
\Phi(T)=\sum \Phi\left(L r_{C} M\right), \quad C \subset \mathfrak{M},
$$

for every $T \in \mathcal{T}$ and every monotone-light factorization of $T$. In interpreting this formula the same agreements as those stated in 4.1 are understood. Using the notation of 2.7 , let $\tau$ be a generic notation for a mapping in $\Delta(T)$. Then (1) can be written in the form

$$
\Phi(T)=\sum \Phi(\tau), \quad \tau \in \Delta(T) .
$$

5.2. Theorem. Assume that $P$ satisfies the condition that every monotone image of $P$ possesses the property $\lambda$ (see 3.8) and that the functional $\Phi$ satisfies the conditions (a), (b), (c) of 1.6. If $\Phi$ is weakly additive in the sense of 5.1, then $\Phi$ is also strongly additive in the sense of 4.1 .

Proof. By the theorem in 4.2 we must show that $\Phi$ satisfies the conditions (d) of 1.6 and (e) of 4.2 .

We first show that $\Phi$ satisfies condition (d) of 1.6. Let $T_{1}, T_{2}$ be mappings in $\mathcal{T}$ which constitute a partition of a mapping $T \in \mathcal{T}$ (see 1.2). Since $\Phi$ is weakly additive, by 5.1 (2) we have

$$
\begin{array}{rlrl}
\Phi(T) & =\sum \Phi(\tau), & & \tau \in \Delta(T) . \\
\Phi\left(T_{1}\right)=\sum \Phi(\tau), & & \tau \in \Delta\left(T_{1}\right) . \\
\Phi\left(T_{2}\right)=\sum \Phi(\tau), & & \tau \in \Delta\left(T_{2}\right) .
\end{array}
$$

Since the middle space $\mathfrak{M}$ in a monotone-light factorization of $T$ is a monotone image of $P, \mathfrak{M}$ possesses the property $\lambda$. By the theorem in 3.11

$$
\Delta(T)=\Delta\left(T_{1}\right)+\Delta\left(T_{2}\right)
$$

(1), (2), (3), and (4) imply that $\Phi(T)=\Phi\left(T_{1}\right)+\Phi\left(T_{2}\right)$.

We now show that $\Phi$ satisfies the condition (e) of 4.2. The middle-space $\mathfrak{M}$ in a monotone-light factorization of a mapping $T \in \mathcal{T}$ possesses the property $\lambda$ by assumption. If a mapping $T$ admits of an unrestricted factorization in which the middle-space is a simple arc, then, by the theorem in $3.14, \mathfrak{M}$ is dendrite. Hence $\Phi(T)=0$. 
5.3. We proceed to show that the theorem of 5.2 applies if $P$ is any unicoherent Peano space (see LA I.2.24). We first give some lemmas needed for this purpose.

Lemma 1. Let $P$ be a unicoherent Peano space and let $X$ be a proper subcontinuum of $P$. Then, for each component $G$ of $P-X$, the frontier of $G$ is a continuum or a point.

Proof. Let $\operatorname{fr}(G)$ designate the frontier of $G$. It follows readily that $G+\mathrm{fr}(G)$ and $P-G$ are continua. Hence $\mathrm{fr}(G)=(G+\mathrm{fr}(G))(P-G)$ is a continuum or a point, since $P$ is unicoherent. $\lambda$.

Lemma 2. Let $P$ be a unicoherent Peano space. Then $P$ possesses the property

Proof. Let $C$ be any proper cyclic element of $P$. Then $C$ is a monotone image of $P$ (see LA II.2.50, II.2.42) and hence $C$ is a unicoherent Peano space (see LA II.1.7). Let $E$ be a closed and totally disconnected subset of $C$. Assume that $C-E$ is not a connected set. Let $G_{0}$ be a component of $C-E$. Then $X=G_{0}+\operatorname{fr}\left(G_{0}\right)$ is a proper subcontinuum of $C-E$. Let $G$ be a component of $C-X$. By Lemma 1 of this section $\mathrm{fr}(G)$ is a continuum or a point. Hence, since $\operatorname{fr}(G) \subset \operatorname{fr}\left(G_{0}\right) \subset E$, fr $(G)$ is a single point. Then $C-\mathrm{fr}(G)$ is not a connected set. This contradicts the assumption that $C$ is a proper cyclic element of $P$. Therefore $P$ possesses the property $\lambda$.

5.4. Lemma. If $P$ is a unicoherent Peano space, then every monotone image of $P$ possesses the property $\lambda$.

Proof. This follows from Lemma 2 of 5.3 since the monotone image of a unicoherent Peano space is a unicoherent Peano space (see LA II.1.7).

5.5. Theorem. Let $P$ be a unicoherent Peano space, and let $\Phi$ be a functional that satisfies the conditions (a), (b), (c) of 1.6 and is weakly additive in the sense of 5.1. Then $\Phi$ is strongly additive in the sense of 4.1 .

Proof. This is a direct consequence of 5.4 and 5.2.

5.6. The strong additivity of the Lebesgue area $A$ (see Introduction) follows now immediately from the facts that (1) $A$ is known to be weakly additive and (2) the square is unicoherent. However, our results apply also in the 2 -sphere case (the case of $F$-surfaces of the type of the 2 -sphere, see LA II.3.24, LA II.3.43). Indeed, $A$ is known to be weakly additive in the 2 -sphere case also (see Youngs [3]), and the 2-sphere is also unicoherent. Thus the theorem of 5.5 shows that the Lebesgue area $A$ is strongly additive in the 2-sphere case also.

5.7. The question arises as to what is the scope of the theorem in 5.2. This is equivalent to asking the following question. For what Peano spaces $P$ is it true that every monotone image of $P$ possess the property $\lambda$ ? The answer 
to this question is given in the following result.

Lemma. In order that every monotone image of a Peano space $P$ possesses the property $\lambda$ it is necessary and sufficient that $P$ be unicoherent.

Proof. If a Peano space $P$ is unicoherent then by 5.4 every monotone image of $P$ possesses the property $\lambda$. This proves the sufficiency. To show the necessity, assume that $P$ is not unicoherent. Then there is a monotone mapping from $P$ onto a Peano space $\mathfrak{M}$ where $\mathfrak{M}$ is cyclic but where $\mathfrak{M}$ contains two points $\mathfrak{x}_{1}, \mathfrak{x}_{2}$ such that $\mathfrak{M}-\left(\mathfrak{x}_{1}+\mathfrak{x}_{2}\right)$ is not connected (see Hall and Youngs [5, Theorem 15]). Thus this particular monotone image of $P$ does not possess the property $\lambda$.

\section{BIBLIOGRAPHY}

LA. T. Rado, Length and area, Amer. Math. Soc. Colloquium Publications, vol. 30, 1948.

1. G. T. Whyburn, Analytic topology, Amer. Math. Soc. Colloquium Publications, vol. 28, 1942.

2. C. B. Morrey, The topology of (path) surfaces, Amer. J. Math. vol. 57 (1935) pp. 17-50.

3. J. W. T. Youngs, The topological theory of Fréchet surfaces, Ann. of Math. vol. 45 (1944) pp. 753-785.

4. T. Rado, On continuous mappings of Peano spaces, Trans. Amer. Math. Soc. vol. 58 (1945) pp. 420-454.

5. D. W. Hall and J. W. T. Youngs, Comments on the cores of certain classes of spaces, Ann. of Math. vol. 48 (1947) pp. 710-716.

The Ohio State University, Columbus, OHo. 